\title{
Pro-CAD: una alternativa para profesionalizar al personal auxiliar del odontólogo.
}

\author{
Pro-CAD «A professionalizing alternative \\ for the dentist's auxiliary staff».
}

\author{
María Teresa González Kokke,* Edna Carmina González Guevara ${ }^{\ddagger}$
}

\section{RESUMEN}

El presente artículo es el resultado de una investigación orientada a la implantación de un modelo educativo, que sustenta una propuesta para la capacitación de recursos humanos de apoyo técnico en el campo de la salud. Para este propósito se denominaron: Asistentes Dentales a quienes son el personal auxiliar que se desempeña con profesionales que ejercen la odontología en forma privada en México, y que no cuentan con escuelas para su formación, considerando sus diferencias individuales e involucrando las áreas cognoscitiva (conocimientos), psicomotriz (habilidades y destrezas) y afectiva (comportamientos). Los recursos humanos compiten fuertemente por encontrar un espacio de desempeño en un mercado altamente exigente, razón por la cual el eje principal de la investigación se fundamentó en el planteamiento del Consejo Normalizador y Certificador de Competencias Laborales (CONOCER). El modelo fue diseñado durante la formación doctoral, enmarcado en la línea de innovación curricular para la calidad total como consecuencia del interés personal por evaluar las competencias del personal auxiliar que se desempeña apoyando al gremio de la odontología; la población está organizada en un colegio local de profesionales filial a la Asociación Dental Mexicana. Se utilizó el método mixto, y la herramienta para recolección de datos fue la encuesta, misma que aportó información relevante para construir una propuesta denominada modelo Pro-CAD (Programa de Capacitación Asistente Dental), que se incluye brevemente al final de este artículo.

Palabras clave: Innovación curricular, personal auxiliar, competencias laborales, práctica odontológica, modelo de capacitación.

\section{ABSTRACT}

This article is the result of research aimed at the implementation of an educational model, which supports a proposal for the training of human resources of technical support in the field of health. For this purpose they were called: Dental Assistants, who are the auxiliary staff who work with professionals who practice dentistry privately in México, and who do not have schools for their training considering their individual differences and involving the cognitive areas (knowledge), psychomotor (skills and skills), affective (behaviors). Human resources compete strongly to find a performance space in a highly demanding market, which is why the main focus of the research was based on the approach of the Standardizing Council and Labour Skills Certificater (CONOCER). The model was designed during my doctoral training framed in the line of curriculum innovation for total quality as a result of the personal interest in evaluating the work skills of the auxiliary staff who perform supporting the dental guild, the population is organized in a local college of professionals affiliated with the Mexican Dental Association. The mixed method was used, and the data collection tool was the survey, which provided relevant information to build a proposal called the Pro-CAD (Dental Assistant Training Program) model that is briefly included at the end of this article.

Keywords: Curriculum innovation, auxiliary staff, work skills, dental practice, training model.

\section{WWV.medigraphic.org.mx}

\footnotetext{
* Cirujano Dentista Universidad Veracruzana. Doctorado en Educación. Universidad de Xalapa UX. México.

‡ Cirujano Dentista Universidad Veracruzana. Especialidad en Salud Pública Xalapa, Ver. México.

Recibido: 02 de noviembre de 2020. Aceptado: 30 de enero de 2021

Citar como: González KMT, González GEC. Pro-CAD: una alternativa para profesionalizar al personal auxiliar del odontólogo. Rev ADM. 2021; 78 (1): 33-41. https://dx.doi.org/10.35366/98385
} 


\section{INTRODUCCIÓN}

E un mundo globalizado, donde las exigencias de calidad son cada vez mayores, surge la necesidad de que los individuos y las instituciones se vayan adaptando a los constantes cambios que conlleva el desarrollo social. La educación ocupa un lugar importante en esta evolución, pues constituye el fundamento esencial que permite al ser humano desarrollarse para transformar su entorno.

En este contexto, el ejercicio de la práctica profesional de la disciplina odontológica no es la excepción, pues el trabajo del cirujano dentista se complementa con el apoyo del personal auxiliar, el cual debe contar con el conocimiento y la capacidad necesarios para lograr la ejecución adecuada de una gran variedad de tratamientos contra las principales patologías que afectan la cavidad oral.

Un consultorio dental se considera una empresa que proporciona servicios de salud y oferta tratamientos a clientes (pacientes). Esto le condiciona al profesional titular, de acuerdo con la normatividad vigente, la responsabilidad de capacitar a los recursos humanos involucrados en las distintas actividades a fin de lograr un desempeño satisfactorio que asegure la calidad. No se puede imaginar a una empresa médica-odontológica trabajando con eficiencia y eficacia si no cuenta con personal asistente suficiente y bien preparado.

Con base en ello, al examinar los nuevos retos en materia de capacitación de los recursos humanos en el campo de la salud, se hace necesario estudiar los cambios que se tienen que implementar al interior de las empresas de servicios odontológicos que mejor puedan apoyar el aprendizaje del trabajador, aportándole elementos para ser cualificado en el mercado laboral.

En ese sentido, se presenta una alternativa de calidad para aquellas personas que no tienen acceso a la educación superior y que se desempeñan laboralmente auxiliando al profesional de la odontología, pero sin una preparación educativa formal. La propuesta responde a la necesidad de resolver el problema de la escasa oferta de programas educativos dirigidos a este recurso humano en el país.

Autores como López, Otero y Blatchford coinciden en la necesidad de ofertar programas de capacitación específicos para el personal que se desempeña como auxiliar en consultorios odontológicos, en donde reciban la debida instrucción para ser realmente de gran ayuda al facultativo y tener la posibilidad de asegurar la calidad a los usuarios del servicio de salud que se brinda. ${ }^{1-3}$

El profesional no podrá alcanzar sus metas de excelencia técnica y científica si no tiene quién complemente su trabajo y le permita proyectarse adecuadamente en el lugar donde se desempeñe.

Por otra parte, las cifras sobre la cobertura de servicios educativos de formación profesional en el país, desafortunadamente, no son muy alentadoras. Según la cita de una nota periodística de Poy, Adolfo Rodríguez Gallardo, académico de la UNAM, dijo claramente en 2009 que los países con las tasas más altas de analfabetismo tienen pocas expectativas de avance.

La sociedad no puede progresar cuando parte de sus integrantes son de los 774 millones de personas que son analfabetas en el mundo, dos terceras partes son mujeres que no han tenido acceso a la instrucción formal; son marginados, no saben leer y no tienen acceso a la educación. ${ }^{4}$

La segregación respecto a la educación está vinculada con la desigualdad de género. Por lo que respecta a México, cifras oficiales del Consejo Nacional de Población (CONAPO), en la misma nota de Poy, revelan que hay 6 millones de personas que no saben leer ni escribir, lo que representa poco más de $8 \%$ de la población; en tanto que 33 millones de mexicanos mayores de 15 años no han logrado concluir su educación básica. ${ }^{4}$

Esto se ve reflejado en un estado como Veracruz, entre otros, donde las instituciones de educación superior están haciendo esfuerzos encarecidos por aumentar la cobertura de la oferta educativa para atender la gran demanda de los jóvenes que buscan acceder a la universidad.

De acuerdo con la experiencia de más de una década de trabajar con el tema y la capacitación de recursos humanos de apoyo al cirujano dentista para algunos grupos colegiados de la región, las autoras detectaron un área de oportunidad: la implantación de programas educativos de formación para el trabajo en el campo de la salud, que persigan, ante todo, el desarrollo personal.

Se parte de la pregunta: ies necesario crear propuestas curriculares para la formación de los recursos humanos que auxilian el ejercicio de la profesión odontológica? El propósito del estudio se centró en el diseño y aplicación de un modelo de capacitación para el personal auxiliar al servicio de la odontología.

En México, el proceso de instrucción, capacitación y formación del personal de apoyo que se desempeña como asistente dental en clínicas o consultorios públicos o privados, se lleva a cabo en forma empírica. Lo anterior tiene su origen en dos factores:

1. El desempeño de esta ocupación no está regulada por las instancias legales, sanitarias, capacitadoras y profesionales, tampoco por la Ley General de Salud del Estado de Veracruz de 2014, en relación con el 
responsable de brindar la capacitación, ya que no se halla dicha disposición en el Título Cuarto, Capítulo I «Disposiciones comunes. Recursos humanos para los servicios de salud», Art. 75 y 77; Capítulo III «Formación, capacitación y actualización de los prestadores de servicios de salud», Art. 83 (p. 35). ${ }^{5}$

2. Existe escasa oferta educativa de programas de calidad para este recurso humano que cualifiquen las competencias necesarias de un técnico que complemente la práctica odontológica profesional.

En todo el país sólo se ofertan talleres y cursos en forma esporádica, ya que por usos y costumbres, el asistente dental es adiestrado regularmente por el odontólogo a cargo, cuya formación disciplinar no aporta a este recurso humano herramientas docentes ni administrativas necesarias para su adecuado desempeño, lo que origina problemas para alcanzar la calidad en el servicio de salud que se brinda.

El modelo de capacitación se diseñó pensando en los profesionales que fungen como empleadores de personal técnico, a los encargados de la capacitación en las empresas dentales medianas y pequeñas, a instructores, consultores de recursos humanos y facilitadores de organismos de capacitación, así como a los propios aspirantes a la formación técnica.

\section{Importancia de la investigación}

Agustín Ibarra refiere que la pertinencia es la correspondencia de los contenidos educativos que se exigen en el mundo laboral, y que es uno de los principales problemas que enfrenta desde siempre el sector educativo. Como solución a esta problemática, se presenta la educación basada en competencias, la cual sirve de referente para el diseño de una currícula adecuada como la que se persiguió en este trabajo. ${ }^{6}$

En este orden de ideas, la relevancia de éste puede resumirse en tres aspectos:

1. En el campo administrativo, un consultorio dental se considera una empresa de servicio, por lo que siempre deberá contar con personal convenientemente entrenado para brindar atención de calidad.

El desempeño humano en toda organización se ve fuertemente influenciado por las condiciones personales y laborales que son medidas a través de la capacitación. Por ello, a los empleadores de asistentes dentales se les aportarán herramientas para selec- cionar, dirigir y capacitar a los recursos humanos de apoyo; quienes ya cuentan con personal capacitado, quizás no deleguen acciones correctas que les faciliten su desempeño y que eviten riesgos para la salud y disminución del índice de urgencias y complicaciones en el consultorio médico. Además, tener a un recurso humano capacitado, les permitirá cumplir con las normas oficiales vigentes en el estado que se ubiquen.

2. En el campo social, el modelo representó una oportunidad de insertarse en el mercado laboral de forma rápida para aquellos jóvenes que no tienen acceso a la educación superior; además, contribuyó al aseguramiento de la calidad de los servicios dentales, pues los usuarios se sienten seguros por la atención de calidad que reciben cuando son atendidos por personal asistente avalado y reconocido oficialmente.

3. En el campo normativo, el modelo tiene la posibilidad de replicarse en otros grupos con colaboradores de profesionales asociados en otros colegios de profesionales del campo de la salud en el país, por lo que representa un área de oportunidad para los organismos dedicados a la regulación de actividades laborales no regladas en la actualidad.

Los conceptos que se manejaron han sido propuestos por diferentes organismos internacionales como UNESCO 1995, OCDE 1997, con su programa de definición y desarrollo de competencias (DeSeCo), el proyecto Tuning de la Unión Europea, la Propuesta para América Latina 2004, así como el proyecto Consejo de Normalización y Certificación de Competencias Laborales en México (CONOCER). ${ }^{7}$

Finalmente, se presentó una alternativa de solución partiendo de las fortalezas y debilidades que se han presentado, así como de la necesidad de desarrollar un enfoque integral y dinámico de formación profesional.

Pro-CAD, como producto final, se justifica con el argumento del trabajo de Díaz, que a partir de una construcción teórica construye una acción educativa para resolver problemas concretos ajustados a las necesidades de cada contexto. ${ }^{8}$

\section{MATERIAL Y MÉTODOS}

La investigación estuvo orientada a la implantación, debido a que se buscó solucionar un problema en torno al tema eje, para lo cual la metodología empleada tuvo un corte mixto, ya que, de acuerdo con Johnson (citado en Hernández, Fernández y Baptista), representa un conjunto de procesos sistemáticos, empíricos y críticos de investigación 
e implica la recolección y análisis de los datos cuantitativos y cualitativos, así como su integración y discusión conjunta para realizar meta-inferencias. ${ }^{9}$ Se tomó como referente importante las aportaciones de Edel, por estar orientadas a la implantación, definida como un conjunto de conocimientos que hacen posible la introducción de nuevos procesos que permitan mejorar los existentes y contribuir a la generación de un proyecto innovador, para el propósito de este trabajo en la disciplina odontológica. ${ }^{10} \mathrm{El}$ autor citado sugiere dos apartados importantes en cualquier investigación para orientar la implantación:

1. El diseño preliminar

2. La propuesta

El primero se construyó a partir de determinar las necesidades de la situación, de tal manera que se pueda llenar ese vacío de información, condición o proceso. Con base en lo que describe Edel, se inició con la jerarquización de las prioridades de la población afectada y se realizó un análisis de sus fortalezas y debilidades organizacionales, de tal modo que se evidenció la problemática de estudio. Se estimaron aquí la situación deseada y la observada, que se pueden apreciar en la Tabla 1. La técnica que se utilizó fue la encuesta, tomando como referencia el texto de Peñal, y el instrumento que se aplicó, el cuestionario. ${ }^{11}$ Validez y confiabilidad del instrumento. Para calcular la consistencia interna del cuestionario, se usó el indicador de fidelidad KR20 de Kuder y Richardson, quienes desarrollaron un procedimiento basado en los resultados obtenidos con cada ítem y que está orientado a instrumentos con ítems dicotómicos, es decir, en los cuales sólo existen dos opciones de respuesta: correcta e incorrecta $\left(1=\right.$ Sí y $0=$ No). ${ }^{12}$ Después de haber aplicado los cuestionarios a asistentes, empleadores y usuarios del servicio dental, se llevó a cabo un piloteo de 12 cuestionarios para cada uno, con 20, 17 y nueve variables, respectivamente. Se procedió a validar el cuestionario con el KR20, el cual arrojó un valor de 0.88 y 0.89 para el de asistentes y usuarios del servicio, lo cual indicó que los instrumentos tuvieron una confiabilidad bastante adecuada. La operalización de las variables se

\section{Tabla 1: Información obtenida en la fase exploratoria del estudio.}

Fortalezas

El Colegio de Cirujanos Dentistas de Xalapa A.C. está constituido legalmente desde 1969 en la Coordinación de Profesiones de la Secretaría de Educación del Estado de Veracruz. Es el primer colegio registrado ante las instancias gubernamentales y está facultado para vigilar el ejercicio de la práctica odontológica en el entorno

El organismo tiene la experiencia de impartir talleres de educación continua para profesionistas y colaboradores

Los profesores que imparten las conferencias tienen práctica privada, lo que les permite vivenciar el problema que se pretende resolver

Existe una estrecha vinculación con la Facultad de Odontología de la Universidad Veracruzana campus Xalapa, en el sentido de hacer uso de las instalaciones y equipo para pilotear el programa de capacitación
Debilidades

Ausencia de programas formales de capacitación para asistentes dentales que se desempeñan laboralmente con los miembros asociados

Desarrollo de competencias laborales sin evaluación ni control interno y externo

La escasa o nula formación pedagógica de los empleadores de asistentes dentales provoca un aprendizaje empírico y práctico por parte de este personal

La movilidad de este personal es alta y la permanencia en el puesto es baja

Los servicios dentales no pueden alcanzar sus niveles de eficiencia, en tanto que el personal de apoyo del profesional no esté debidamente entrenado para desempeñar el puesto

No existen documentos orientadores para la capacitación de este personal

Fuente: elaboración propia. 


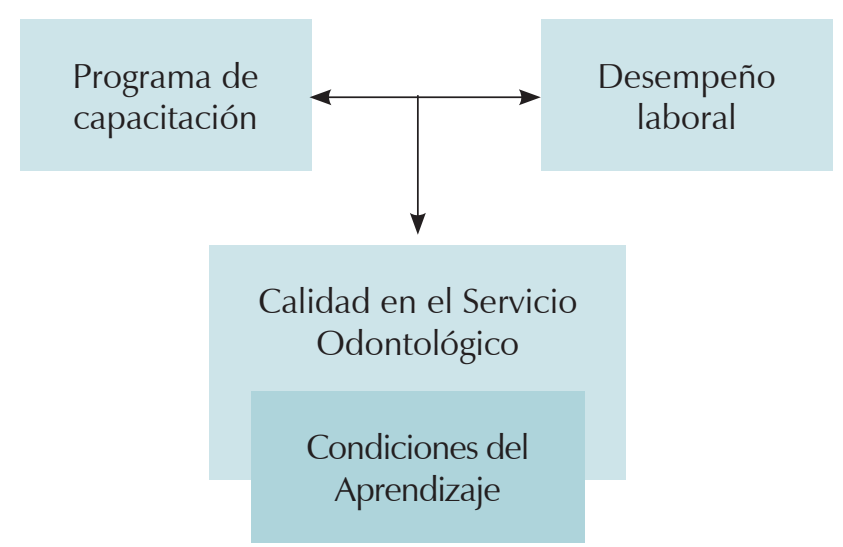

Figura 1: Operacionalización de variables.

muestra en la Figura 1. El alcance fue aplicativo, porque se intervino en forma educativa manipulando la variable objeto de estudio y aportando los elementos para alcanzar el objetivo general: evaluar las competencias de este colectivo antes y después de intervenir en forma educativa mediante el programa denominado Pro-CAD.

La población de estudio se obtuvo de la selección intencionada de asistentes dentales que se desempeñan en empresas del sector privado en Xalapa, Veracruz. Auxiliando a profesionales asociados en un organismo colegiado que se organiza a partir de 1964, Colegio de Cirujanos Dentistas de Xalapa A.C., filial de la Asociación Dental Mexicana, Federación Nacional de Colegios de Cirujanos Dentistas, que cuentan con un certificado vigente de la profesión.

\section{RESULTADOS}

Se realizaron dos fases: la exploratoria en donde por medio de la encuesta se concentra la triangulación de información obtenida de asistentes dentales, empleadores y usuarios del servicio dental. Y la segunda fase consistió en la intervención educativa mediante el Pro-CAD.

Resultados exploratorios del cuestionario para asistentes dentales. La población total bajo estudio fue de 49 asistentes dentales, de los cuales tres fueron hombres y 46 mujeres. El tiempo de trabajar como asistentes dentales varió de menor a cinco años a mayor de 16 años, siendo la media de 3.90 y la mediana de 2 .

Respecto a la distribución porcentual con base en la edad, indica que la mitad de la población capacitada tiene entre 15 y 19 años de edad, (siendo éste el rango que presenta mayor frecuencia de la población bajo estudio), seguida de $25 \%$ de la población que tiene de 20 a 24 años de edad, mientras que $16.7 \%$ de los asistentes cuentan con una edad mayor a 30 años, y sólo 8.3\% entre 25 y 29.

En cuanto a la distribución porcentual según el tiempo de trabajar como asistentes dentales, indica que 33\% de la población capacitada tiene dos años laborando como asistente dental, (siendo ésta la duración con mayor frecuencia), siguiéndole con $25 \%$ la población capacitada que tiene menor o igual a un año trabajando como asistente, de igual manera, los que tienen una antigüedad de tres años, mientras que la población de cuatro y cinco años laborando como asistente sólo cuenta con 8.3\%, para cada rango.

La capacitación con mayor frecuencia la hacen directamente sus jefes, con $35.29 \%$, cursos con $29.41 \%$, mientras que la mitad de $35.3 \%$ cursó parte de la carrera de odontología y la otra mitad la concluyó, y sólo a la mitad, $23.52 \%$ de la población de los asistentes dentistas, le enseñó un compañero de trabajo, y la otra mitad aprendió observando.

En la Figura 2 se aprecia claramente que los aspectos considerados indispensables para ser asistente dental son presentación y actitud, con 31 y $27 \%$, respectivamente; no obstante, se tiene considerado que los aspectos más importantes a la hora de buscar este empleo es la aspiración salarial y conocimientos, con un 80 y 73\%; cabe mencionar que la edad y la experiencia son considerados como elementos que rara vez importan, con 24 y $20 \%$, respectivamente.

La intervención profesional más frecuente, realizada por más de la mitad de la población bajo estudio, es la odontología general, con un $61.22 \%$, seguida de la or-



Figura 2: Análisis de correspondencias múltiple de las variables del tipo capacitación y actividades realizadas en el trabajo. 


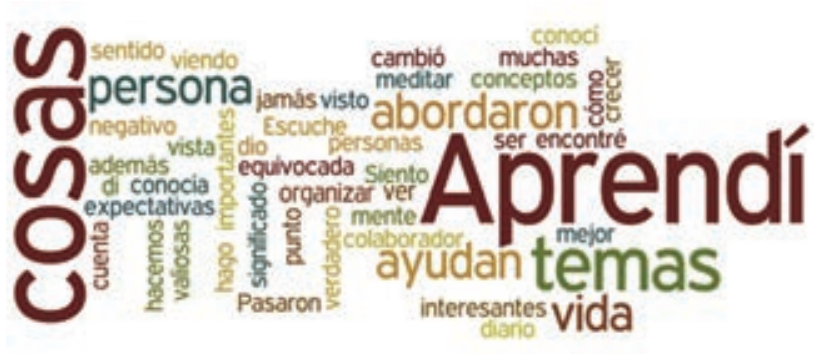

Figura 3: Nube de palabras que escenifica lo que ocurrió durante la capacitación Pro-CAD.

todoncia con $14.29 \%$, el $12.24 \%$ de la población realiza intervenciones como un tratamiento endodóncico o una rehabilitación protésica; además, los tratamientos de periodoncia y odontología geriátrica no son recurridos en esta población.

La mayor razón por la que emplean personal en el consultorio es para tener efectividad en el servicio (83.67\%), mientras que $8.16 \%$ de los empleadores tienen personal por costumbre, y sólo $4.08 \%$ de la población bajo estudio lo hace porque trabaja poco o por comienzo.

Los aspectos considerados indispensables para contratar un asistente dental es la presentación, seguida de la aspiración salarial, con sólo 37 y 18\%, respectivamente; sin embargo, se tiene considerado que lo más importante a la hora de contratar un asistente son la aspiración salarial, la escolaridad y la presentación (76, 67 y 51\%, respectivamente); cabe mencionar que la edad y la experiencia no se consideran importantes, con $69 \%$ cada uno, teniendo a las recomendaciones con un $45 \%$, que rara vez importa.

Por lo general, un integrante es el que maneja todas las funciones, con excepción del registro fotográfico, esta función la suele llevar a cabo el integrante dos, con 78\% de la población.

En cuanto al análisis funcional, los aspectos considerados más importantes para los usuarios es el trato del personal hacia los pacientes y la presentación del dentista, ambos con un porcentaje del 92\%, siguiéndole la experiencia de un odontólogo con 88\%, también consideran importante el costo de los tratamientos con $82 \%$.

Resultados antes y después de la capacitación ProCAD. La población total bajo estudio fue de 12 asistentes dentales, que fueron capacitados mediante el modelo Pro-CAD, de los cuales 11 fueron mujeres y sólo un hombre. El tiempo de trabajar como asistentes dentales varió de menor o igual a un año a cinco años, siendo la media de 2.41 y la mediana de 2 . Con respecto a la distribución porcentual con base en el tiempo de trabajar como asistentes dentales, indica que 33\% de la población capacitada tiene dos años laborando como asistente dental, (siendo ésta la duración con mayor frecuencia), siguiéndole con $25 \%$ la población capacitada que tiene menor o igual a un año trabajando como asistente, de igual manera, los que tienen una antigüedad de tres años, mientras que la población de cuatro y cinco años laborando como asistente sólo cuenta con 8.3\% para cada rango, uno de los motivos más representativos para asistir a la capacitación del Pro-CAD, es que tuvieron deseos de aprender más sobre la odontología y ser mejores en su trabajo como asistentes dentales.

Resultados del instrumento evaluador del modelo de capacitación Pro-CAD, aplicado a los asistentes del curso después de intervenir 211 horas de capacitación: en la figuras 3 y 4 se muestra la nube de palabras que se utilizaron con mayor frecuencia para esta variable fueron ser y responsable, siguiéndole por disciplinado y valorarme, lo que significa que después de tomar la capacitación Pro-CAD, los asistentes se visualizan siendo más responsables, colaborando con su jefe con mayor confianza y entendiendo mejor los temas en el trabajo.

\section{DISCUSIÓN}

\section{Reflexión sobre los hallazgos en la investigación en la primera fase exploratoria}

El resultado de la etapa exploratoria arrojó información que permitió acercarnos a dimensionar la naturaleza del problema planteado al inicio de la investigación: la ausencia de programas educativos para asistentes dentales. Entre los hallazgos más sobresalientes se encontró que:



Figura 4: Nube de palabras que escenifica la visualización de los asistentes dentales después de la capacitación Pro-CAD. 




Figura 5: Egresadas del Pro-CAD.

- Existe una baja permanencia en la ocupación de asistente dental: el tiempo promedio es de dos años.

- En su mayoría, los asistentes dentales se han formado en la práctica misma al margen de un proceso pedagógico.

- La relación entre el auxiliar y el profesional de la odontología es muy cercana; en su mayoría, se trata de binomios de acción que aprenden sobre la misma práctica.

- La primera persona que contacta a los pacientes es el asistente dental. Su campo de acción es polivalente y fuerte influencia sobre la empresa (consultorio dental).

- El problema más frecuente para el desempeño del asistente dental es la carencia de capacitación formal y de estímulo económico por actividades extra. Sin embargo, llama la atención que su nivel de compromiso bajo le es atribuido a la falta de interés por servir, como resultado de que no percibe un reconocimiento por parte del equipo de atención.

- Los pacientes prefieren recibir tratamientos dentales en lugares donde los reciban con calidez y el servicio sea eficiente.

- El personal manifiesta su deseo de superación y la posibilidad de ir progresando en su preparación para alcanzar mejores oportunidades y logros personales.

Esta información nos permitió realizar un diagnóstico que puede resumirse con base en una serie de necesidades que nos dieron la pauta para la planeación del piloto del modelo de capacitación:
- La necesidad del profesional de la odontología de contar con personal auxiliar capacitado.

- La necesidad de un grupo poblacional de ser capacitado en un programa de calidad.

- La exigencia disciplinar de tener un programa guía formal para la capacitación de su personal.

- La necesidad de profesionalizar al personal auxiliar.

- La necesidad de que un organismo valide las competencias disciplinarias y profesionales del recurso humano referido.

- La necesidad social de tener cursos de capacitación que se obtengan a través de un modelo formal que posibilite el reconocimiento social y disciplinar.

Con respecto a la distribución porcentual según la edad, se indica que la mitad de la población capacitada tiene entre 15 y 19 años de edad, (siendo éste el rango que presenta mayor frecuencia de la población bajo estudio), siguiéndole el $25 \%$ de la población que tiene de 20 a 24 años de edad, mientras que $16.7 \%$ de los asistentes cuentan con una edad mayor o igual a 30 años, y sólo $8.3 \%$ cuenta con una edad de entre 25 y 29 .

\section{Reflexión sobre los hallazgos mediante la aplicación del piloto del modelo de capacitación Pro-CAD}

Con base en la información obtenida en la primera etapa, se tomó como punto de partida las necesidades de capacitación con la finalidad de fundamentar el programa pedagógico, el cual se basó en las competencias profesionales y cuyos contenidos están estructurados de tal forma que proporcionan los elementos necesarios para el desempeño adecuado del asistente dental.

Cabe mencionar que, de acuerdo con el instrumento de evaluación del aprovechamiento, se rebasaron las expectativas que se tenían de la intervención educativa. Esto ocurrió en tres aspectos principalmente:

1. La participación de los aspirantes a asistentes dentales en el proyecto impactó positivamente en el aspecto actitudinal de este grupo poblacional, ya que manifestaron sentirse reconocidos por sus jefes y por su familia.

2. Entre los temas abordados, el de mayor aceptación fue el relacionado con el desarrollo humano, contrario a la lógica de inclinarse por algún procedimiento técnico disciplinar en particular.

3. Los profesores especialistas que participaron en la aplicación de Pro-CAD evaluaron el nivel de apro- 


\section{Perfiles}

\section{Perfil social}

Personas preferentemente con bachillerato terminado, con o sin experiencia, responsables, que deseen laborar en la atención de las necesidades del cirujano dentista de práctica general $y / o$ especialista en el consultorio dental, brindando tratamientos para la preservación de la salud bucal a nivel público o privado
Módulos profesionales

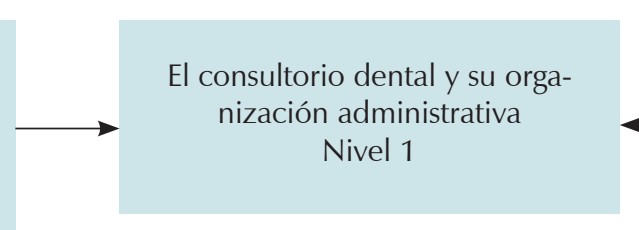

consultorio dental y su orgaNivel 1

\section{Unidades de competencia}

Preparar los materiales, procesar la información de la consulta dental y cuidar las condiciones sanitarias del entorno en las áreas de su competencia

\section{Perfil profesional}

Personal capacitado para reconocer $y$ entender conceptos, procesos, sistemas e información de la disciplina odontológica, con habilidad y criterio adecuado para la ejecución de tareas específicas orientadas a eficientar el trabajo auxiliando al odontológo

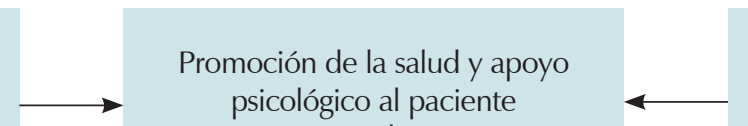

Nivel 2
Colaborar en la prestación de cuidados al paciente aplicando a su nivel de técnicas de apoyo y educación sanitaria

\section{Perfil institucional}

Asistentes dentales como una formación integral de calidad De acuerdo con la misión, visión e ideario de la institución que avala el modelo Pro-CAD

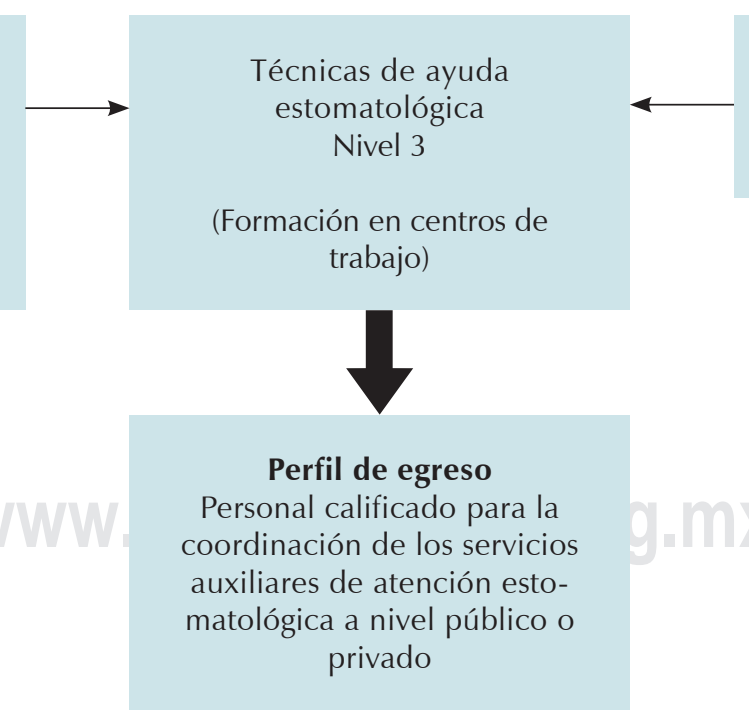

Realizar tareas de instrumentación eficiente junto al profesional

Fuente: elaboración propia.

Figura 6: Relación de los niveles de competencia con los módulos de capacitación. 
vechamiento de los asistentes como exitoso con base en el diagnóstico de cada una de sus intervenciones, las cuales se registraron mediante rúbricas.

\section{CONCLUSIONES}

Los recursos humanos auxiliares al servicio de la odontología no deben continuar desempeñándose al margen de la oportunidad de capacitarse dentro de un proceso formal de calidad que responda a las necesidades de la población afectada (profesionales de la odontología, pacientes, y los propios recursos: asistentes dentales). Con lo que se responde afirmativamente a la pregunta de investigación planteada.

Los supuestos hipotéticos se probaron mediante la aplicación del piloto obteniendo mejora del desempeño del grupo poblacional estudiado en cuatro aspectos: calidad, rendimiento, eficiencia y confiabilidad.

Se logró alcanzar los objetivos planteados con el diseño y aplicación del modelo Pro-CAD (Figura 5). El cual puede definirse así:

Pro-CAD es un modelo de capacitación socialmente pertinente, diseñado bajo el enfoque de competencias en la categoría de formación para el trabajo de asistente dental desde una perspectiva multidisciplinar, que surgió para ayudar a resolver un problema de índole educativo. Lo cual puede calificarse con tres atributos: original, innovador y exitoso (Figura 6).

\section{AGRADECIMIENTOS}

Nuestro agradecimiento especial a los socios de la ADM que fungieron como maestros en la capacitación durante el piloto, así como sus aportes para la realización de este trabajo de investigación.

\section{REFERENCIAS}

1. López E, Chaparro M. Competencias laborales del trabajador vistas desde el mercado laboral. Parte A. Revista Tabula Rasa. 2006; (5). Colombia: Universidad Colegio Mayor de Cundimarca. Recuperado de: www.redalyc.org 39600514.

2. Otero J. Asistencia odontológica. Curso online Gerencia y Marketing Dental. Lima, Perú. 2011. Recuperado el 02 de octubre de 2011, Disponible en: www. odontomarketing.com.

3. Blatchford B. Editorial [Mensaje en un blog]. 2011. Recuperado de: http://blatchford.com/.

4. Poy L. Mujeres sin acceso a la educación formal, $66 \%$ de los analfabetas en el mundo. Periódico La Jornada. 2009. Recuperado de: http://www.jornada.unam.mx/2009/04/09/sociedad/037n3soc

5. Secretaría de Gobierno del Estado de Veracruz. Ley de Salud del Estado de Veracruz de Ignacio de La Llave. Edición virtual. 2014. Recuperado de http://www.uv.mx/saisuv/files/2014/08/Ley-deSalud-del-Estado-de-Veracruz.pdf.

6. Ibarra AE. Certificación de competencia laboral: Nuevo paradigma, nuevos retos para la población laboral y educativa. Simposio llevado a cabo en la Reunión de Ministros de Educación de las Américas en Punta del Este, departamento de Maldonado. 2001.

7. Organización Internacional del Trabajo. Clasificaciones de ocupaciones, competencias y formación profesional: Paralelismo o convergencias ASAE. 2003. Recuperado de http://www.asaenet. org/copyright/,1937, html.

8. Díaz AF. Metodología de diseño curricular para la educación superior. México: Trillas, 2012.

9. Hernández R, Fernández C, Baptista P. Metodología de la investigación. México: McGrawHill, 2014.

10. Edel R. Diseño de proyectos de Investigación en Ciencias Sociales y Humanidades. México: Universidad Veracruzana, Plaza y Valdés. 2007.

11. Peñal D. Análisis de datos multivariantes. Granada, España: McGraw-Hill, 2002.

12. Urbina O. Metodología para la evaluación de competencias laborales en salud. Revista Cubana de Salud Pública, 2010; 36 (2): Consultado en: www.redalyc.org. 2141613501.

\section{Correspondencia: \\ Dra. Ma Teresa González Kokke \\ E-mail: teresagonzalez@uv.mx}

Conflicto de intereses: Los autores declaran no tener ningún conflicto de intereses. 